Document downloaded from:

http://hdl.handle.net/10251/64197

This paper must be cited as:

Casabán Bartual, MC.; Cortés López, JC.; Romero Bauset, JV.; Roselló Ferragud, MD. (2015). Probabilistic solution of random SI-type epidemiological models using the Random Variable Transformation technique. Communications in Nonlinear Science and Numerical Simulation. 24(1):86-97. doi:10.1016/j.cnsns.2014.12.016.

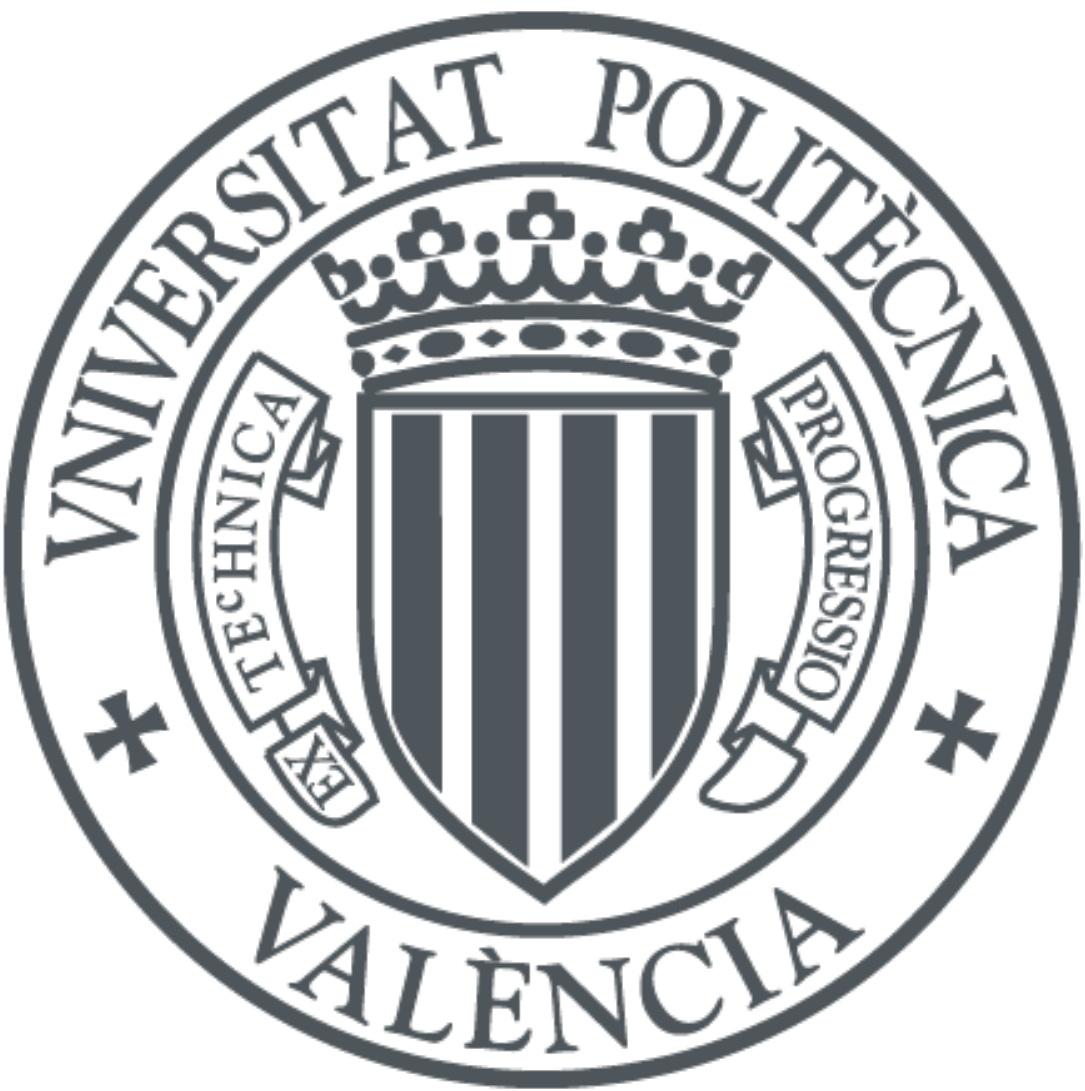

The final publication is available at

https://dx.doi.org/10.1016/j.cnsns.2014.12.016

Copyright Elsevier

Additional Information 


\title{
Probabilistic solution of random SI-type epidemiological models using the Random Variable Transformation technique
}

\author{
M.-C. Casabán a,*, J.-C. Cortés ${ }^{\mathrm{a}}$, J.-V. Romero ${ }^{\mathrm{a}}$, M.-D. Rosellóa \\ ${ }^{a}$ Instituto Universitario de Matemática Multidisciplinar, \\ Universitat Politècnica de València, \\ Camino de Vera s/n, 46022, Valencia, Spain
}

\begin{abstract}
This paper presents a full probabilistic description of the solution of random SI-type epidemiological models which are based on nonlinear differential equations. This description consists of determining: the first probability density function of the solution in terms of the density functions of the diffusion coefficient and the initial condition, which are assumed to be independent random variables; the expectation and variance functions of the solution as well as confidence intervals and, finally, the distribution of time until a given proportion of susceptibles remains in the population. The obtained formulas are general since they are valid regardless the probability distributions assigned to the random inputs. We also present a pair of illustrative examples including in one of them the application of the theoretical results to model the diffusion of a technology using real data.
\end{abstract}

Keywords: Random SI-type epidemiological models, first-order nonlinear random differential equations, Random Variable Transformation technique, first probability density function

\section{Motivation}

Due to the non-deterministic nature of phenomena that appear in areas like epidemiology, biology, engineering, physics, chemistry, social sciences, the mathematical description of such phenomena is often modelled by random/stochastic differential equations. This approach is supported because of input parameters (coefficients, forcing terms, initial and/or boundary conditions) that appear in such equations have specific real interpretations but their values are not known with certainty. Examples of such situations are the contagion coefficient in epidemiology, the diffusion coefficient in thermodynamics, the coefficient of viscosity in fluid mechanics, the propagation coefficient in the theory of wave propagation, etc. In a first approach, these mathematical equations can be treated as deterministic using, as representative value of each input parameter, the average value of a set of sampled observations experimentally obtained. This can be done in specific problems as those ones which admit repeated trials in laboratories or environments under control. Things become more complicated when only one single sample is available

\footnotetext{
${ }^{*}$ Corresponding author

Email addresses: macabar@imm.upv.es (M.-C. Casabán), jccortes@imm.upv.es (J.-C. Cortés), jvromero@imm.upv.es (J.-V. Romero), drosello@imm.upv.es (M.-D. Roselló) 
as often happens in epidemiology or social sciences. Even in the former case where the experiment can be performed repeatedly, then the mean values found could vary. If this variation is large, the mean value actually used may be non-representative. This motivates the consideration of input parameters as random variables (r.v.'s) or stochastic processes (s.p.'s) rather than deterministic constants or functions, respectively. It is thus advantageous to consider the equations that describe such models as being random rather than deterministic.

In dealing with differential equations with uncertainty, it is important to point out that two classes of equations are possible, namely, stochastic differential equations (s.d.e.'s) and random differential equations (r.d.e.'s). Both types of differential equations are distinctly different and they require completely different techniques for analysis and approximation. On the one hand, s.d.e.'s are forced by an irregular process such as a Wiener process or Brownian motion. S.d.e.'s are typically written in terms of stochastic differentials, but they are interpreted as Itô or Stratonovich stochastic integrals $[1,2]$. On the other hand, r.d.e.'s are those in which random effects are manifested directly in input parameters which are assumed to have specific probability distributions such as uniform, beta, exponential, gamma, etc. [3,4]. Throughout this paper r.d.e.'s will be considered only. This is motivated because in our model the initial condition represents a rate lying in the interval $[0,1]$ and beta distribution is a flexible and appropriate choice to model it.

Solutions of r.d.e.'s are s.p.'s, say $X(t ; \omega)$, rather than classical functions. Usually, time variable $t$ lies in $[0, \infty[$ and $\omega \in \Omega$, where $(\Omega, \widetilde{F}, \mathbb{P})$ is a probability space. For simplicity, the $\omega$-parameter is hidden and the s.p. is denoted by $X(t)$. In contrast to what happens in dealing with deterministic differential equations, where the main aim is computing the solution, in dealing with r.d.e.'s the computation of the mean, $\mathbb{E}[X(t)]$, and the variance, $\mathbb{V}[X(t)]$, is also a goal in itself. Furthermore, a more desirable goal is to compute the first probability distribution (1-p.d.f.), say $f_{1}(x, t)$, of $X(t)$ since from it one can determine all its statistical moments

$$
\mathbb{E}\left[(X(t))^{n}\right]=\int_{-\infty}^{\infty} x^{n} f_{1}(x, t) \mathrm{d} x, \quad n=0,1,2, \ldots
$$

In particular, using (1) the mean and variance can be obtained straightforwardly. Further important information such as the probability that $X(t)$ lies in specific intervals of interest or its quantiles can also be obtained by $f_{1}(x, t)$.

Random Variable Transformation (R.V.T.) method is a powerful technique to compute the p.d.f. of a r.v. which results from the mapping of another r.v. [3, p.21-25], [5]. In the context of differential and difference equations, R.V.T. method has been recently applied in order to determine the 1-p.d.f. of random ordinary differential equations [6,7], random partial differential equations $[8,9,10,11]$ and random difference equations [12]. In [13], a number of illustrative examples show that R.V.T. technique can still be useful when only approximations of the solution s.p. are available.

In this paper, we are interested in applying R.V.T. technique to study a class of random nonlinear models that mainly appear in epidemiology to model a type of communicable diseases, usually referred to as SI-type models. This kind of models are useful to study simple epidemics where the population consists of individuals that are either susceptibles (S) or infected (I). In its basic formulation, it is assumed that the total population size, say $n$, is constant for all time $t$. This hypothesis is plausible during certain time-intervals particularly in developed countries as well as for populations under control. SI-models can be stated through the following initial value 
problem (i.v.p.)

$$
\left\{\begin{array}{l}
S^{\prime}(t)=-\frac{\beta}{n} S(t)[n-S(t)], \quad t>0, \\
S(0)=m,
\end{array}\right.
$$

where, $S(t)$ denotes the number of susceptibles at the time instant $t, m$ represents the number of susceptibles at the beginning (that corresponds to $t=0$ ) over the total population size $n$, and $\beta>0$ denotes the rate of decline in the number of susceptibles. Illustrative examples where deterministic and random SI-models are applied in different biological contexts can be found in $[14,15,16,17,18]$.

In [19], authors present a random SI-model where the unknown $S(t)$ is rewritten in terms of the proportion of susceptibles at time $t, P(t)=S(t) / n$, which leads the following i.v.p.

$$
\left\{\begin{array}{l}
P^{\prime}(t)=-\beta P(t)[1-P(t)], \quad t>0, \\
P(0)=P_{0},
\end{array}\right.
$$

where $P_{0}=m / n \in[0,1]$. In this manner, as the initial condition is assumed to be a r.v., authors suppose throughout the paper that $P_{0}$ has a beta distribution whose domain is the interval $[0,1]$. For simplicity, the nature of the positive parameter $\beta>0$ is assumed to be deterministic. In a first step, authors establish a number of theoretical results which are applied to model the prevalence of people with HIV antibodies in a representative sample of homosexual men.

Apart from epidemiology, SI-model encounters fruitful applications in many different frameworks since the population of infected is not restricted to the spread of diseases but it can also be interpreted as the population that, for instance, acquires a new technology. In this context, the susceptibles population represents potential customers being the remainder of the population who have already adopted the new technology [20,21].

In this paper we will deal with the random SI-model (3), where uncertainty is considered in both, the initial condition $P_{0}$ and the rate of decline in the proportion of susceptibles $\beta$. To conduct this study we will take advantage of the R.V.T. method. In order to provide more generality to our analysis, in a first stage, we will permit that both positive random inputs $P_{0}$ and $\beta$ have any probability distribution. In a second stage, we will illustrate by means of two examples the theoretical results established. In particular, we will model the evolution of the user penetration rate of mobile phone lines in Spain over the last few years.

The paper is organized as follows, in Section 2, we introduce a number of results related to R.V.T. method that will be required throughout the paper. Section 3 is devoted to compute the 1-p.d.f. and the mean and variance functions of the solution s.p. of the SI-model (3). Then, using this information, confidence intervals are also included. This section ends by providing a general formula to compute the distribution of time until a given proportion of susceptibles remains in the population, which is an information of great interest in applications. In Section 4, a pair of examples illustrate the theoretical results developed in Section 3. Conclusions are drawn in the last section.

\section{Preliminaries}

For the sake of clarity in the presentation, in this section we introduce a number of results that will be used throughout this paper.

The Random Variable Transformation method (R.V.T.) is a probability technique that permits to calculate the p.d.f., $f_{Y}(y)$, of a r.v. $Y$ which comes from mapping another r.v., $X$, whose p.d.f. is known. The scalar version of R.V.T. method establishes that if: 
- $X$ is an absolutely continuous r.v. on the domain $D_{X}=\left\{x: x_{1} \leq x \leq x_{2}\right\}$,

- $f_{X}(x)$ denotes the p.d.f. of r.v. $X$,

- $r: D_{X} \subseteq \mathbb{R} \longrightarrow \mathbb{R}$ is a monotone increasing (decreasing) mapping that transforms r.v. $X$ into r.v. $Y$, i.e., $Y=r(X)$, and

- there exists the inverse function of mapping $r, s(y)=x$, which has continuous derivative on the domain $D_{Y}=\left\{y: y_{1}=r\left(x_{1}\right) \leq y \leq r\left(x_{2}\right)=y_{2}\right\}\left(D_{Y}=\left\{y: y_{1}=r\left(x_{2}\right) \leq y \leq r\left(x_{1}\right)=y_{2}\right\}\right)$,

then, the p.d.f. of r.v. $Y$ is given by

$$
f_{Y}(y)=f_{X}(s(y))\left|s^{\prime}(y)\right|, \quad y \in D_{Y} .
$$

A generalization of the scalar R.V.T. method can be found in $[5,22]$. It consists of splitting the domain of r.v. $X$ into a number of subintervals where the mapping $r$ is monotone and then applying (4) in each piece.

Now, we establish two results that will be used later to study the SI-model (3). They will be obtained by applying R.V.T. formula (4) for particular cases of mapping $r$.

Proposition 1 (R.V.T. technique: inverse-vertical translation transformation). Let $c \in \mathbb{R}$ and $X$ be an absolutely continuous real r.v. defined on a probability space $(\Omega, \mathfrak{F}, \mathbb{P})$, with p.d.f. $f_{X}(x)$. Assume that $X$ is a non-zero r.v. and let us denote by $D_{X}$ the domain of r.v. $X$, where

$$
D_{X}=I_{x}^{-} \cup I_{x}^{+}, \quad\left\{\begin{array}{l}
I_{x}^{-}=\{x=X(\omega) \in \mathbb{R}:-\infty<x<0, \omega \in \Omega\}, \\
I_{x}^{+}=\{x=X(\omega) \in \mathbb{R}: 0<x<+\infty, \omega \in \Omega\} .
\end{array}\right.
$$

Then, the p.d.f. $f_{Y}(y)$ of the inverse-vertical translation transformation $Y=\frac{1}{X}+c$ is given by

$$
f_{Y}(y)=\frac{1}{(y-c)^{2}} f_{X}\left(\frac{1}{y-c}\right), \quad y \in D_{Y}=I_{y}^{-} \cup I_{y}^{+}, \quad\left\{\begin{array}{l}
I_{y}^{-}=\{y \in \mathbb{R}: y<c\}, \\
I_{y}^{+}=\{y \in \mathbb{R}: y>c\} .
\end{array}\right.
$$

Proof Let us consider the mapping $y=r(x)=\frac{1}{x}+c$, that is monotone in each subinterval $-\infty<x<0$ and $0<x<+\infty$. So, its inverse function exists and takes the form

$$
x=s(y)=\frac{1}{y-c},
$$

being

$$
s^{\prime}(y)=-\frac{1}{(y-c)^{2}},
$$

its first derivative. Then, by applying (4) in each subinterval and taking into account (6)-(7), one gets expression (5). Note that the computation of the domain $D_{Y}$ follows straightforwardly since the transformation $r(x)$ is decreasing monotone in each subinterval. 
Proposition 2 (R.V.T. technique: inverse-horizontal translation transformation). Let $d \in \mathbb{R}$ and $X$ be an absolutely continuous real r.v. defined on a probability space $(\Omega, \mathfrak{F}, \mathbb{P})$, with p.d.f. $f_{X}(x)$. Assume that $X-d$ is a non-zero r.v. and let us denote by $D_{X}$ the domain of r.v. $X$, where

$$
D_{X}=I_{x}^{-} \cup I_{x}^{+}, \quad\left\{\begin{array}{l}
I_{x}^{-}=\{x=X(\omega) \in \mathbb{R}:-\infty<x<d, \omega \in \Omega\}, \\
I_{x}^{+}=\{x=X(\omega) \in \mathbb{R}: d<x<+\infty, \omega \in \Omega\} .
\end{array}\right.
$$

Then, the p.d.f. $f_{Y}(y)$ of the inverse-horizontal translation transformation $Y=\frac{1}{X-d}$ is given by

$$
f_{Y}(y)=\frac{1}{y^{2}} f_{X}\left(\frac{1}{y}+d\right), \quad y \in D_{Y}=I_{y}^{-} \cup I_{y}^{+}, \quad\left\{\begin{array}{l}
I_{y}^{-}=\{y \in \mathbb{R}: y<0\}, \\
I_{y}^{+}=\{y \in \mathbb{R}: y>0\} .
\end{array}\right.
$$

Proof Let us consider the monotone mapping $y=r(x)=\frac{1}{x-d}$, that is monotone in each subinterval $-\infty<x<d$ and $d<x<+\infty$. So, its inverse function exists and takes the form

$$
x=s(y)=\frac{1}{y}+d
$$

being

$$
s^{\prime}(y)=-\frac{1}{y^{2}},
$$

its first derivative. Then, by applying (4) to each subinterval and taking into account (9)-(10), one gets the expression (8). The computation of the domain $D_{Y}$ follows straightforwardly since the transformation $r(x)$ is decreasing monotone in each subinterval.

Later, the following generalization of R.V.T. technique in two dimensions [5, 22] will be needed.

Theorem 1. Let $\mathbf{X}=\left(X_{1}, X_{2}\right)$ be a two-dimensional r.v. with joint p.d.f. $f_{X_{1}, X_{2}}\left(x_{1}, x_{2}\right)$. Let $\mathbf{Y}=$ $\left(Y_{1}=r_{1}\left(X_{1}, X_{2}\right), Y_{2}=r_{2}\left(X_{1}, X_{2}\right)\right)$ be a two-dimensional r.v. where $y_{1}=r_{1}\left(x_{1}, x_{2}\right), y_{2}=r_{2}\left(x_{1}, x_{2}\right)$ is a bijective continuous mapping whose inverse is given by $x_{1}=s_{1}\left(y_{1}, y_{2}\right), x_{2}=s_{2}\left(y_{1}, y_{2}\right)$. Let us assume that the partial derivatives $\frac{\partial x_{1}}{\partial y_{1}}, \frac{\partial x_{1}}{\partial y_{2}}, \frac{\partial x_{2}}{\partial y_{1}}$ and $\frac{\partial x_{2}}{\partial y_{2}}$ are continuous and the jacobian $J=\frac{\partial x_{1}}{\partial y_{1}} \frac{\partial x_{2}}{\partial y_{2}}-\frac{\partial x_{2}}{\partial y_{1}} \frac{\partial x_{1}}{\partial y_{2}} \neq 0$. Then, the joint p.d.f. $f_{Y_{1}, Y_{2}}\left(y_{1}, y_{2}\right)$ of the random vector $\mathbf{Y}=\left(Y_{1}, Y_{2}\right)$ is given by

$$
f_{Y_{1}, Y_{2}}\left(y_{1}, y_{2}\right)=f_{X_{1}, X_{2}}\left(s_{1}\left(y_{1}, y_{2}\right), s_{2}\left(y_{1}, y_{2}\right)\right)|J| .
$$

We close this section stating a result that will be crucial later. It has been recently established in [7] and it provides a comprehensive study to compute the 1-p.d.f. of the solution s.p. to the following random linear i.v.p.

$$
\left.\begin{array}{rl}
\dot{Z}(t) & =A Z(t)+B, \quad t>t_{0} \\
Z\left(t_{0}\right) & =Z_{0}
\end{array}\right\}
$$

where inputs $Z_{0}, B$ and $A$ are assumed to be distinct r.v.'s. In our subsequent analysis just the following particular case of (12), that corresponds to $B=0$ with probability 1 , will be required. 
Proposition 3 ([7], Case I.3). Let us consider the linear random i.v.p.

$$
\left.\begin{array}{rl}
\dot{Z}(t) & =A Z(t), \quad t>t_{0} \\
Z\left(t_{0}\right) & =Z_{0}
\end{array}\right\}
$$

where inputs $Z_{0}$ and $A$ are assumed to be absolutely continuous r.v.'s on a common probability space $(\Omega, \mathcal{F}, \mathbb{P})$. Let us denote by

$$
\begin{aligned}
D_{Z_{0}} & =\left\{z_{0}=Z_{0}(\omega), \omega \in \Omega: z_{0,1} \leq z_{0} \leq z_{0,2}\right\}, \\
D_{A} & =\left\{a=A(\omega), \omega \in \Omega: a_{1} \leq a \leq a_{2}\right\},
\end{aligned}
$$

their respective domains and $f_{Z_{0}, A}\left(z_{0}, a\right)$ their joint p.d.f. Then, the 1-p.d.f. of the solution s.p. $Z(t)$ of (13) is given by

$$
f_{1}(z, t)=\frac{1}{t-t_{0}} \int_{\mathrm{e}^{a_{1}\left(t-t_{0}\right)}}^{\mathrm{e}^{a_{2}\left(t-t_{0}\right)}} f_{Z_{0}, A}\left(\frac{z}{\xi}, \frac{\ln (\xi)}{t-t_{0}}\right) \frac{1}{\xi^{2}} \mathrm{~d} \xi, \quad z_{1} \leq z \leq z_{2}, \quad \forall t>t_{0},
$$

where

$$
\begin{array}{lll}
z_{1}=z_{0,1} \mathrm{e}^{a_{1}\left(t-t_{0}\right)}, & z_{2}=z_{0,2} \mathrm{e}^{a_{2}\left(t-t_{0}\right)}, & \text { if } \quad z_{0,1}>0, \\
z_{1}=z_{0,1} \mathrm{e}^{a_{2}\left(t-t_{0}\right)}, & z_{2}=z_{0,2} \mathrm{e}^{a_{2}\left(t-t_{0}\right)}, & \text { if } \quad z_{0,1} z_{0,2} \leq 0, \\
z_{1}=z_{0,1} \mathrm{e}^{a_{2}\left(t-t_{0}\right)}, & z_{2}=z_{0,2} \mathrm{e}^{a_{1}\left(t-t_{0}\right)}, & \text { if } \quad z_{0,2}<0 .
\end{array}
$$

If $t=t_{0}$,

$$
f_{1}\left(z_{0}, t_{0}\right)=\int_{a_{1}}^{a_{2}} f_{Z_{0}, A}\left(z_{0}, a\right) \mathrm{d} a, \quad z_{0,1} \leq z_{0} \leq z_{0,2}
$$

\section{Computing the 1-p.d.f. and further statistical information to the random SI-model}

In this section we will determine important statistical information associated to the solution s.p., $P(t)$ of the random SI-model (3), namely, the 1-p.d.f. and the expectation and variance functions. Based on these quantities, we will be able to construct confidence intervals for $P(t)$ at any fixed confidence level. Finally, we will obtain the p.d.f. of the time until a given proportion of susceptibles remains in the population. This function provides epidemiologists crucial information that helps them, for example, to determine whether a disease is under control or it could become a pandemic.

\subsection{First probability density function (1-p.d.f.)}

This section is devoted to compute a probabilistic solution of the nonlinear SI-model (3) where the inputs parameters, $P_{0}$ and $\beta$, are assumed to be independent positive r.v.'s, being

$$
\begin{aligned}
D_{P_{0}} & =\left\{p_{0}=P_{0}(\omega), \omega \in \Omega: 0 \leq p_{0,1} \leq p_{0} \leq p_{0,2} \leq 1\right\}, \\
D_{\beta} & =\left\{\beta=\beta(\omega), \omega \in \Omega: 0 \leq \beta_{1}<\beta<\beta_{2}\right\},
\end{aligned}
$$

their respective domains. Hereinafter, $f_{P_{0}}\left(p_{0}\right)$ and $f_{\beta}(\beta)$ will denote their p.d.f.'s, respectively. Notice that in contrast to the SI-model studied in [19], we permit the contagion parameter $\beta$ be random. This assumption is realistic since numerous physical, genetic, environmental, etc., factors, which usually involve uncertainty, are naturally embedded in $\beta$.

In order to obtain the probabilistic solution of i.v.p. (3), i.e., the 1-p.d.f. of the solution s.p. $P(t)$ of i.v.p. (3), in a first step we will transform (3) into a linear i.v.p. that adapts to expression 
(12) and then, the resulting i.v.p. will be again transformed to be better identified with model (13). Therefore the results established in Proposition 3 will be applicable. To this end, let us first consider the change of variable

$$
Q(t)=\frac{1}{P(t)}, \quad t>0,
$$

then, the problem (3) can be recast as

$$
\begin{aligned}
Q^{\prime}(t) & =\beta Q(t)-\beta, \quad t>0, \\
Q(0) & =\frac{1}{P_{0}} .
\end{aligned}
$$

Note that, regarding the i.v.p. (12), we have taken $t_{0}=0$. Nevertheless, r.d.e. (19) does not fit yet the pattern (12) since the random diffusion coefficient $A$ and the random forcing term $B$ must be distinct. To accommodate equation (19) to model (12), we introduce a new change of variable

$$
H(t)=Q(t)-1
$$

In this manner, the i.v.p. (19)-(20) reads

$$
\begin{aligned}
H^{\prime}(t) & =\beta H(t), \quad t>0, \\
H(0) & =\frac{1}{P_{0}}-1,
\end{aligned}
$$

which corresponds to the i.v.p. (13) with the identification:

$$
Z_{0}=\frac{1}{P_{0}}-1, A=\beta, Z(t)=H(t), t_{0}=0 .
$$

As the p.d.f. $f_{P_{0}}\left(p_{0}\right)$ of the random input $P_{0}$ is assumed to be known, the p.d.f. of the random initial condition $Z_{0}$ defined in (24) can be computed by Proposition 1, and hence we can take advantage of the results established in Proposition 3. So, for $t>0$ fixed, the application of formula (15) permits us to compute the p.d.f. of the r.v. $H=H(t)$, which corresponds to the solution s.p. $H(t)$ of the i.v.p. (22)-(23) evaluated at that $t>0$

$$
f_{H}(h)=\frac{1}{t} \int_{e^{a_{1} t}}^{e^{a_{2} t}} f_{Z_{0}, A}\left(\frac{h}{\xi}, \frac{\ln (\xi)}{t}\right) \frac{1}{\xi^{2}} \mathrm{~d} \xi .
$$

Taking into account (24) and (17) for the computation of the domain of integration and that $P_{0}$ and $\beta$ are assumed to be independent r.v.'s, (25) can be written as

$$
f_{H}(h)=\frac{1}{t} \int_{e^{a_{1} t}}^{e^{a_{2} t}} f_{Z_{0}}\left(\frac{h}{\xi}\right) f_{A}\left(\frac{\ln (\xi)}{t}\right) \frac{1}{\xi^{2}} \mathrm{~d} \xi=\frac{1}{t} \int_{e^{\beta_{1} t}}^{e^{\beta_{2} t}} f_{P_{0}}\left(\frac{\xi}{h+\xi}\right) f_{\beta}\left(\frac{\ln (\xi)}{t}\right) \frac{1}{(h+\xi)^{2}} \mathrm{~d} \xi,
$$

where in the last step Proposition 1 has been applied to $X=P_{0}, Y=Z_{0}$ and $c=-1$.

To conclude, we need to compute the p.d.f. $f_{P}(p)$ of r.v. $P=P(t)$, being $t>0$ arbitrary but fixed. This can be done taking into account that by (18) and (21)

$$
\begin{gathered}
P(t)=\frac{1}{H(t)+1}, \\
7
\end{gathered}
$$


for each $t>0$, and applying Proposition 2 to $X=H, Y=P, d=-1$ and $f_{X}(x)=f_{H}(h)$, where $f_{H}(h)$ is given by (26). This yields

$$
f_{P}(p)=\frac{1}{p^{2}} f_{H}\left(\frac{1}{p}-1\right)=\frac{1}{t} \int_{e^{\beta_{1} t}}^{e^{\beta_{2} t}} f_{P_{0}}\left(\frac{p \xi}{1-p+p \xi}\right) f_{\beta}\left(\frac{\ln (\xi)}{t}\right) \frac{1}{(1-p+p \xi)^{2}} \mathrm{~d} \xi .
$$

As the previous development holds for every $t>0$, we conclude that the 1-p.d.f. of the solution s.p. $P(t)$ of the i.v.p. (3) is given by

$$
f_{1}(p, t)=\frac{1}{t} \int_{e^{\beta_{1} t}}^{\beta^{\beta_{2} t}} f_{P_{0}}\left(\frac{p \xi}{1-p+p \xi}\right) f_{\beta}\left(\frac{\ln (\xi)}{t}\right) \frac{1}{(1-p+p \xi)^{2}} \mathrm{~d} \xi .
$$

Note that if $t=0, P(0)=P_{0}$ and then $f_{1}(p, 0)$ is just the p.d.f. of the random input $P_{0}$.

Finally, it is important to point out that expression (27) is general, since it holds regardless the specific p.d.f.'s assumed for the model inputs $P_{0}$ and $\beta$. It confers (27) great flexibility from a practical standpoint where a wide range of p.d.f.'s can be considered in order to deal with specific models.

Remark 1. From a practical point of view, in dealing with the SI-type epidemiological model (3), it is natural to assume that the initial condition $P_{0}$ and the rate of decline $\beta$ are independent r.v.'s. However, expression (27) can also be generalized to the case that these input parameters are assumed to be dependent r.v.'s. In fact, let us apply Theorem 1 to $X_{1}=P_{0}, X_{2}=\beta, Y_{1}=Z_{0}$, $Y_{2}=A$ and

$$
\begin{aligned}
& Z_{0}=r_{1}\left(P_{0}, \beta\right)=\frac{1}{P_{0}}-1 \Rightarrow P_{0}=s_{1}\left(Z_{0}, A\right)=\frac{1}{Z_{0}+1}, \\
& A=r_{2}\left(P_{0}, \beta\right)=\beta \Rightarrow \beta=s_{2}\left(Z_{0}, A\right)=A \text {. }
\end{aligned}
$$

Notice that the jacobian of mapping $s\left(Z_{0}, A\right)=\left(s_{1}\left(Z_{0}, A\right), s_{2}\left(Z_{0}, A\right)\right)$ is given by

$$
J=\frac{\partial s_{1}\left(Z_{0}, A\right)}{\partial Z_{0}} \times \frac{\partial s_{2}\left(Z_{0}, A\right)}{\partial A}-\frac{\partial s_{2}\left(Z_{0}, A\right)}{\partial Z_{0}} \times \frac{\partial s_{1}\left(Z_{0}, A\right)}{\partial A}=-\frac{1}{\left(Z_{0}+1\right)^{2}} \neq 0 .
$$

Hence, according to (11) the joint p.d.f. of $\left(Z_{0}, A\right)$ is given by

$$
f_{Z_{0}, A}\left(z_{0}, a\right)=f_{P_{0}, \beta}\left(\frac{1}{z_{0}+1}, a\right) \frac{1}{\left(z_{0}+1\right)^{2}} .
$$

Now, instead of obtaining the p.d.f. $f_{H}(h)$ given by (26) where independence between the inputs $P_{0}$ and $\beta$ has been used, we can obtain an analogous expression in the general case that both r.v.'s are assumed to be dependent with joint p.d.f. $f_{P_{0}, \beta}\left(p_{0}, \beta\right)$. Indeed, by (25) and (28) one gets

$$
f_{H}(h)=\frac{1}{t} \int_{e^{a_{1} t}}^{a^{a_{2} t}} f_{P_{0}, \beta}\left(\frac{\xi}{h+\xi}, \frac{\ln (\xi)}{t}\right) \frac{1}{(h+\xi)^{2}} \mathrm{~d} \xi .
$$

From this expression and following an analogous development as we showed previously, it is straightforwardly to obtain

$$
f_{1}(p, t)=\frac{1}{t} \int_{e^{\beta_{1} t}}^{e^{\beta_{2} t}} f_{P_{0}, \beta}\left(\frac{p \xi}{1-p+p \xi}, \frac{\ln (\xi)}{t}\right) \frac{1}{(1-p+p \xi)^{2}} \mathrm{~d} \xi .
$$

This expression extends (27) in the sense that it holds when $P_{0}$ and $\beta$ are dependent r.v.'s with joint p.d.f. $f_{P_{0}, \beta}\left(p_{0}, \beta\right)$. 


\subsection{Mean, variance and confidence intervals}

In the context of the applications we will consider in the next section, the above expression of $f_{1}(p, t)$ is useful to compute important statistical information associated to $P(t)$ at the time instant $t$ such as, the mean, $\mu_{P}(t)=\mathbb{E}[P(t)]$, and the variance, $\left(\sigma_{P}(t)\right)^{2}=\mathbb{V}[P(t)]$, functions. In agreement with (1), these functions can be computed as follows

$$
\mu_{P}(t)=\mathbb{E}[P(t)]=\int_{-\infty}^{\infty} p f_{1}(p, t) \mathrm{d} p, \quad\left(\sigma_{P}(t)\right)^{2}=\mathbb{V}[P(t)]=\int_{-\infty}^{\infty} p^{2} f_{1}(p, t) \mathrm{d} p-\left(\mu_{P}(t)\right)^{2},
$$

where $f_{1}(p, t)$ is given by (27).

At this point, practical questions concerning the control of the spread of epidemics, the diffusion of new technologies, etc., may be raised. For instance, in what ways are these statistical moments results useful for monitoring the evolution of a disease or the success of an advertising campaign? The answer must hinge upon the criteria chosen for determining when the number of susceptibles (or equivalently, the number of infected) has exceeded preset threshold, say $\lambda>0$. For each $t>0$, the Tchebycheff inequality (see [3, p.19]) together with (31) provide an upper bound on the probabilistic criterion previously chosen

$$
\mathbb{P}\left[\left|P(t)-\mu_{P}(t)\right| \geq \lambda\right] \leq \frac{\left(\sigma_{P}(t)\right)^{2}}{\lambda^{2}} .
$$

However, the computation of the 1-p.d.f., $f_{1}(p, t)$, of the solution s.p. $P(t)=P(t ; \omega)$ permits to specify not just punctual predictions for $P(t)$ as $\mu_{P}(t)$, but also to construct confidence intervals for any $(1-\alpha) \times 100 \%$ confidence level. Indeed, fixed $\alpha \in(0,1)$, for each time instant $t$ one can determine $x_{1}(t)$ and $x_{2}(t)$, such that

$$
1-\alpha=\mathbb{P}\left(\left\{\omega \in \Omega: P(t ; \omega) \in\left[x_{1}(t), x_{2}(t)\right]\right\}\right)=\int_{x_{1}(t)}^{x_{2}(t)} f_{1}(p, t) \mathrm{d} p
$$

and

$$
\int_{0}^{x_{1}(t)} f_{1}(p, t) \mathrm{d} p=\frac{\alpha}{2}=\int_{x_{2}(t)}^{1} f_{1}(p, t) \mathrm{d} p .
$$

In practice, $95 \%$ confidence intervals, which correspond to $\alpha=0.05$, are usually considered.

\subsection{Distribution of time until a given proportion of susceptibles remains in the population}

So far, we have obtained the 1-p.d.f. $f_{1}(p, t)$ for the proportion $P(t)$ of susceptibles at time $t$ (see (27)) and its main statistical information (see (31) and (33)). From a practical standpoint, it is very useful to know when the proportion of susceptibles in the population will reach a specific value. For instance, what is the expected time before twenty percent of the population remains susceptible? To provide an answer to this question, a distribution for r.v. $T$, the time until some proportion $\rho$ of the population remains susceptible, must be defined. Obviously, the answer will depend on the probability distributions to $P_{0}$ (the initial proportion of susceptibles) and $\beta$ (the contagion rate) as well as the target proportion of susceptibles, $\rho$.

In order to determine the distribution of r.v. $T$, let us observe that the exact solution of the nonlinear r.d.e. (3) is given by

$$
P(T)=\frac{P_{0}}{\mathrm{e}^{\beta T}\left(1-P_{0}\right)+P_{0}} .
$$


Then, set $\rho=P(T)$ and solve for $T$ to obtain

$$
T=\frac{1}{\beta} \ln \left(\frac{P_{0}(1-\rho)}{\rho\left(1-P_{0}\right)}\right) .
$$

Now, we apply Theorem 1 to

$$
\begin{array}{llll}
X_{1}=\beta, & Y_{1}=T, & Y_{1}=r_{1}\left(X_{1}, X_{2}\right)=\frac{1}{X_{1}} \ln \left(\frac{X_{2}(1-\rho)}{\rho\left(1-X_{2}\right)}\right), & X_{1}=s_{1}\left(Y_{1}, Y_{2}\right)=\frac{1}{Y_{1}} \ln \left(\frac{Y_{2}(1-\rho)}{\rho\left(1-Y_{2}\right)}\right), \\
X_{2}=P_{0}, & Y_{2}=P_{0}, & Y_{2}=r_{2}\left(X_{1}, X_{2}\right)=X_{2}, & X_{2}=s_{2}\left(Y_{1}, Y_{2}\right)=Y_{2} .
\end{array}
$$

Taking into account that $\frac{\partial s_{2}\left(y_{1}, y_{2}\right)}{\partial y_{1}}=0$, the jacobian is $J=-\frac{1}{\left(y_{1}\right)^{2}} \ln \left(\frac{y_{2}(1-\rho)}{\rho\left(1-y_{2}\right)}\right) \neq 0$, hence the joint p.d.f. of $\left(Y_{1}, Y_{2}\right)=\left(T, P_{0}\right)$ is given by

$$
\begin{aligned}
f_{T, P_{0}}\left(t, p_{0}\right) & =\frac{1}{t^{2}}\left|\ln \left(\frac{p_{0}(1-\rho)}{\rho\left(1-p_{0}\right)}\right)\right| f_{\beta, P_{0}}\left(\frac{1}{t} \ln \left(\frac{p_{0}(1-\rho)}{\rho\left(1-p_{0}\right)}\right), p_{0}\right) \\
& =\frac{1}{t^{2}}\left|\ln \left(\frac{p_{0}(1-\rho)}{\rho\left(1-p_{0}\right)}\right)\right| f_{\beta}\left(\frac{1}{t} \ln \left(\frac{p_{0}(1-\rho)}{\rho\left(1-p_{0}\right)}\right)\right) f_{P_{0}}\left(p_{0}\right),
\end{aligned}
$$

where the independence between r.v.'s $P_{0}$ and $\beta$ has been considered in the last step. Therefore, the $P_{0}$-marginal distribution of $f_{T, P_{0}}\left(t, p_{0}\right)$ yields the p.d.f. of $T$

$$
f_{T}(t ; \rho)=\frac{1}{t^{2}} \int_{\max \left(p_{0,1}, c_{1}\right)}^{\min \left(p_{0,2}, c_{2}\right)}\left|\ln \left(\frac{p_{0}(1-\rho)}{\rho\left(1-p_{0}\right)}\right)\right| f_{\beta}\left(\frac{1}{t} \ln \left(\frac{p_{0}(1-\rho)}{\rho\left(1-p_{0}\right)}\right)\right) f_{P_{0}}\left(p_{0}\right) \mathrm{d} p_{0}, \quad p_{0} \in D_{P_{0}}
$$

where $D_{P_{0}}$ is defined by (17) and

$$
c_{1}=\frac{\rho \mathrm{e}^{\beta_{1} t}}{\rho \mathrm{e}^{\beta_{1} t}+(1-\rho)}, \quad c_{2}=\frac{\rho \mathrm{e}^{\beta_{2} t}}{\rho \mathrm{e}^{\beta_{2} t}+(1-\rho)} .
$$

For $t$ and $\rho$ previously fixed, these values have been determined by imposing that

$$
\beta_{1}<\frac{1}{t} \ln \left(\frac{p_{0}(1-\rho)}{\rho\left(1-p_{0}\right)}\right)<\beta_{2}
$$

being $\beta_{1}$ and $\beta_{2}$ the values that define the domain $D_{\beta}$ given in (17). In (39), $f_{T}(t ; \rho)$ describes the distribution of time until $\rho$ percent of the population is susceptible.

\section{Examples}

In this section, we will illustrate the theoretical results previously obtained through a pair of examples. The aim of the first example is to compute the 1-p.d.f. of the solution s.p. $P(t)$ of the i.v.p. (3) as well as its expectation, variance functions and confidence intervals. In the second example, we show how to apply the i.v.p. (3) in order to model the diffusion of a technology, namely, the user penetration rate of mobile phone lines in Spain over the last few years.

Example 1. Let us consider the i.v.p. (3) where the random initial condition $P_{0}$ is assumed to have a beta distribution of parameters $a=200$ and $b=20$, i.e., $P_{0} \sim \operatorname{Be}(200 ; 20)$ and the nonlinear coefficient $\beta$ has a gamma distribution of parameters $\lambda=1000$ and $\tau=1 / 1000$, i.e., 
$\beta \sim \mathrm{Ga}(1000 ; 1 / 1000)$. We will assume that $P_{0}$ and $\beta$ are independent r.v.'s. Figure 1 shows the 1-p.d.f. of the solution s.p. $P(t)$. It has been computed by expression (27). In Figure 2, we have plotted the expectation $\left(\mu_{P}(t)\right)$ and standard deviation $\left(\sigma_{P}(t)\right)$ of $P(t)$ according to (27)(31). Since $P(t)$ tends to zero as $t$ increases, the expectation and standard deviation functions converge to zero. This behaviour is in agreement with the plot of the 1-p.d.f. showed in Figure 1 where one observes that $f_{1}(p, t)$ concentrates about $p=0$ as times increases. Figure 3 shows the expectation and $95 \%$ confidence intervals, they have been computed according to expressions (33)-(34). Note that confidence intervals about $t=2.5$ become wider what is in agreement with the shape of standard deviation plot shown in Figure 2 (right).

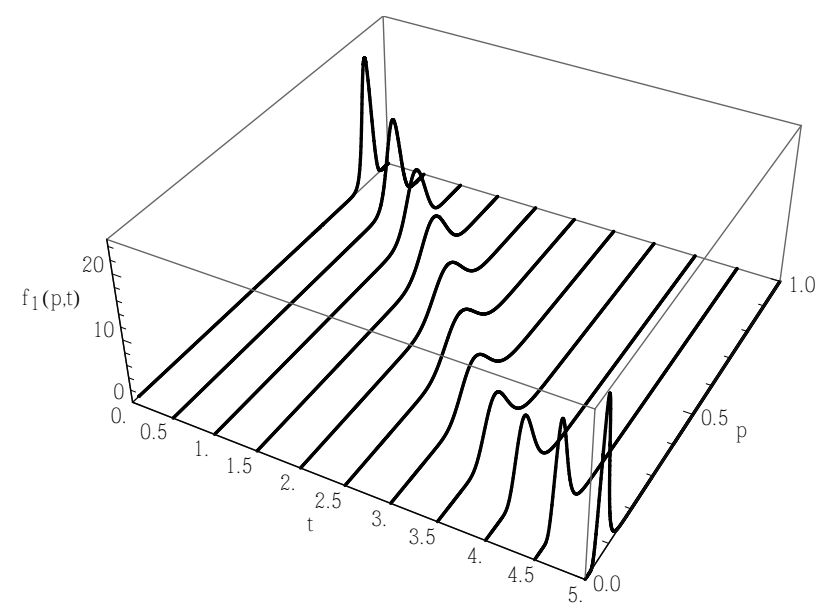

Figure 1: Plot of $f_{1}(p, t)$ given by (27) in Example 1 at different values of $t=\{0,0.1,0.2,0.3, \ldots, 1\}$.
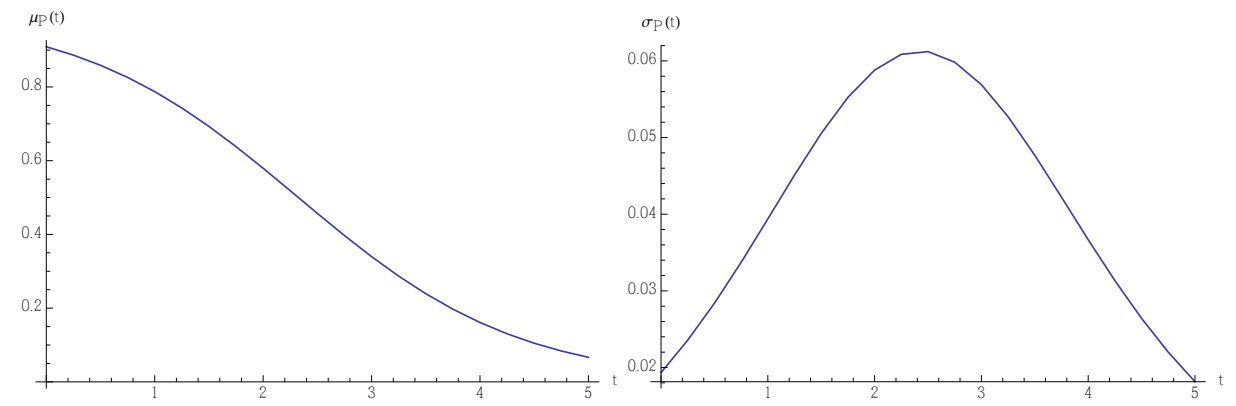

Figure 2: Expectation function $\mu_{P}(t)$ (left) and the standard deviation function $\sigma_{P}(t)$ of $P(t)$ (right) in Example 1.

Example 2. As it has been pointed out in Section 1, the SI-model (3) can be applied to study the diffusion of a new technology. In this example, we will take advantage of the theoretical results previously established to model the user penetration rate of mobile phone lines in Spain. Table 1 collects the available data during the years 1995-2011, see [23]. Hereinafter, $x_{i}$ will 


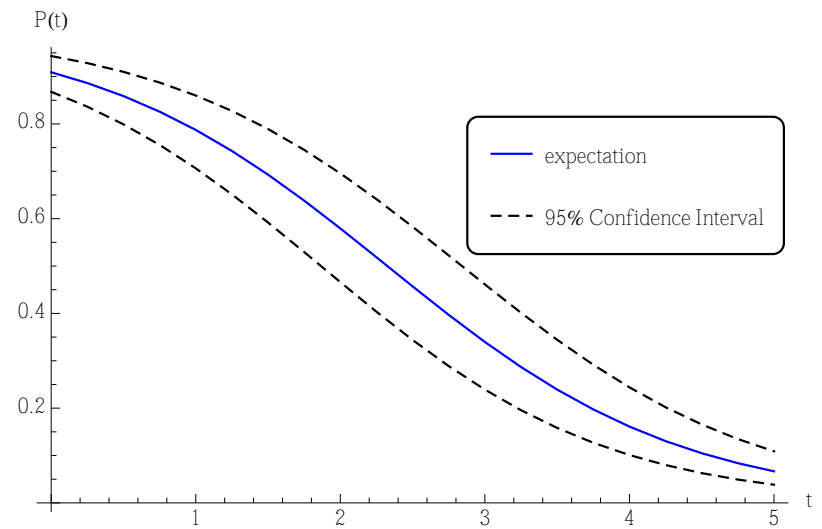

Figure 3: Expectation (solid line) and 95\% confidence intervals (dotted lines) in Example 1.

denote the penetration rate of mobile phone lines in Spain at the year $t_{i}, i=0,1, \ldots, 16$, that correspond to $1995,1996, \ldots, 2011$, respectively. The value $x_{i}$ represents the rate of mobile phone lines per 100 inhabitants taking as reference the Spanish census corresponding to year 2011 updated by INE (National Statistics Institute of Spain). Notice that, the penetration rate, $x_{i}$, may be greater than $100 \%$ since any individual can possess more than one mobile phone line. In order to be able to apply the model (3), two transformations on the data listed in Table 1 will be done. First, we standardize the values $x_{i}$ by assuming a saturation value of 115 . Secondly, as the unknown $P(t)$ of model (3) represents the percentage of susceptibles instead of infected (i.e., the percentage of people who have already adopted the mobile phone technology), we take $P_{i}=1-x_{i} / 115, i=0,1, \ldots, 16$, hence $P_{i} \in(0,1)$. The resulting values $P_{i}$ are given in Table 2. This motivates the choice of a beta distribution of parameters $a, b>0$ for the random initial condition, $P_{0} \sim \operatorname{Be}(a ; b)$. As long as, the diffusion coefficient $\beta$ is assumed to have a gamma distribution of parameters $\lambda, \tau>0$, i.e., $\beta \sim G a(\lambda ; \tau)$. We make this decision because $\beta$ is a positive parameter and gamma distribution is a flexible model which has two degree of freedom whose domain is $(0, \infty)$.

\begin{tabular}{|c|c|c|c|c|c|c|c|c|c|}
\hline year & 1995 & 1996 & 1997 & 1998 & 1999 & 2000 & 2001 & 2002 & 2003 \\
\hline penetration rate $\left(x_{i}\right)$ & 2.3 & 7.5 & 10.2 & 16.2 & 37.3 & 59.9 & 72.6 & 81.9 & 89.3 \\
\hline \hline year & 2004 & 2005 & 2006 & 2007 & 2008 & 2009 & 2010 & 2011 & -- \\
\hline penetration rate $\left(x_{i}\right)$ & 91.2 & 99.2 & 104.4 & 108.9 & 109.6 & 111.4 & 111.7 & 113.9 & -- \\
\hline
\end{tabular}

Table 1: User penetration rates of mobile phone lines in Spain during the years 1995-2011. Source [23]

In order to determine the model parameters $a, b, \lambda$ and $\tau$, we have split the sample data in two pieces. The first one corresponds from year 1995 to $2001\left(t_{0}=1995, t_{1}=1996, \ldots, t_{6}=2001\right)$ and it has been used to adjust the model (3). The rest of the sample data will be used to validate the model. Model fitting has been carried out by minimizing the mean square error between the data $P_{i}$ and the expectation given by (31) evaluated at the time instants $t_{i}, i=0,1, \ldots, 6$. It leads 


\begin{tabular}{|c|c|c|c|c|c|c|c|c|c|}
\hline year & 1995 & 1996 & 1997 & 1998 & 1999 & 2000 & 2001 & 2002 & 2003 \\
\hline$P_{i}$ & 0.9800 & 0.9348 & 0.9113 & 0.8591 & 0.6757 & 0.4791 & 0.3687 & 0.2878 & 0.2235 \\
\hline \hline year & 2004 & 2005 & 2006 & 2007 & 2008 & 2009 & 2010 & 2011 & -- \\
\hline$P_{i}$ & 0.2070 & 0.1374 & 0.0922 & 0.0530 & 0.0470 & 0.0313 & 0.028695 & 0.0096 & -- \\
\hline
\end{tabular}

Table 2: Data collected in Table 1 after their transformation. Source [23]

to the following optimization programme

$$
\min _{a, b, \lambda, \tau>0} E(a, b, \lambda, \tau)=\sum_{i=0}^{6}\left(P_{i}-\mathbb{E}[P(t ; a, b, \lambda, \tau)]\right)^{2},
$$

where, according to (31) and taking into account that $P(t)=P(t ; a, b, \lambda, \tau) \in(0,1)$, the above expectation can be computed as

$$
\mathbb{E}[P(t ; a, b, \lambda, \tau)]=\int_{0}^{1} p f_{1}(p, t) \mathrm{d} p .
$$

Since $P_{0} \sim B e(a ; b)$ and, $\beta \sim G a(\lambda ; \tau)$, hence $\beta \in\left(\beta_{1}, \beta_{2}\right)=(0, \infty)$, the 1-p.d.f. given by (27) reads

$$
f_{1}(p, t)=\frac{1}{t} \int_{1}^{\infty} f_{P_{0}}\left(\frac{p \xi}{1-p+p \xi}\right) f_{\beta}\left(\frac{\ln (\xi)}{t}\right) \frac{1}{(1-p+p \xi)^{2}} \mathrm{~d} \xi,
$$

where

$$
f_{P_{0}}\left(\frac{p \xi}{1-p+p \xi}\right)=\frac{\Gamma(a+b)}{\Gamma(a) \Gamma(b)}\left(\frac{p \xi}{1-p+p \xi}\right)^{a-1}\left(\frac{1-p}{1-p+p \xi}\right)^{b-1}
$$

and

$$
f_{\beta}\left(\frac{\ln (\xi)}{t}\right)=\lambda^{\tau} \xi^{-\frac{\lambda}{t}} \frac{1}{\Gamma(\tau)}\left(\frac{\ln (\xi)}{t}\right)^{\tau-1}
$$

The optimum of the error function (42) has been computed using the NMinimize command by Mathematica software applying the Nelder-Mead algorithm [24]. It provides the following parameters values

$$
a^{*}=114.95, \quad b^{*}=1.83, \quad \lambda^{*}=27.36, \quad \tau^{*}=0.032 .
$$

Figure 4 displays the 1-p.d.f. $f_{1}(p, t)$ given by (44)-(46) for the optimal values $a^{*}, b^{*}, \lambda^{*}$ and $\tau^{*}$ given by (47). For the sake of clarity in its plotting, the range of the vertical axis has been cut off since $f_{1}(p, t)$ takes very large values as $p$ is close to 0 and 1 . In Figure 5 , the mean function (left) and the standard deviation function (right) have been plotted. Taking advantage of expressions (33)-(34), in Figure 6, the expectation function (solid line) together with 95\% confidence intervals have been plotted. In this graphic, the blue points correspond to the years $1995, \ldots, 2001$ used to compute the parameters $a, b, \lambda, \tau$ given by (47) whereas the red points are the rest of the real data corresponding from year 2002 to 2011. Note that all these data lie inside 95\% confidence intervals except the one that corresponds to the year 2010 which hardly exceeds the limit. However, we can observe that the model fitting for the period 2002-2008 is not as accurate as for the rest of the years. It is important to point out that this issue could be expected. 
Indeed, at the beginning when a new technology is released it often generates great expectation and, afterwards, there is often a lack of interest. This fact together with the significant Spanish population increase experienced during the economic boom, corresponding to the period 20022008, may explain that after some years the points are above the average prediction.

From Figure 4, we observe the 1-p.d.f. becomes more platykurtic about $t=2002$. It is in agreement with the shape of the standard deviation function which has a maximum about this year. This graphical behaviour is also observed in Figure 6, where confidence intervals widen about year 2002.

Finally, Figure 7 displays the p.d.f. of the time $T$ until a proportion $\rho$ of susceptibles remains in the population. Expressions (39)-(41) have been used to perform this plot. As $\rho$ goes to 1, the p.d.f. $f_{T}(t ; \rho)$ tends to concentrates about $t=0$ which means that a very few time is required in order to the number of susceptibles being in the population is maximum. This function permits governments or telecommunications companies to provide significant information such as the average time until, for example, a 90\% of the population has not adopted this technology (or conversely, $10 \%$ of the population has acquired this technology), just by computing the following the integral

$$
\mathbb{E}[T]=\int_{0}^{\infty} t f_{T}(t ; 0.90) \mathrm{d} t=2.654 \text { years, }
$$

which is given in terms of the p.d.f. $f_{T}(t ; \rho)$, defined by (39)-(40) for $\rho=0.90$. Notice that this value, 2.654 years, is in agreement with the figures shown in Table 2 since it is between $t=2$ and $t=3$, that correspond to years 1997 and 1998, where the percentages of people who had not yet adopted the technology were $91.13 \%$ and $85.91 \%$, respectively. This means, in time-average, that in August 1998, 90\% of the Spanish population had not yet acquired a mobile phone. In Table 3, we show the figures corresponding to the above expectation, $\mathbb{E}[T]$, for different values of $\rho$.

\begin{tabular}{|c|c|c|c|c|c|c|c|}
\hline$\rho$ & 0.90 & 0.80 & 0.70 & 0.60 & 0.50 & 0.40 & 0.30 \\
\hline $\mathbb{E}[T]$ & 2.654 & 3.616 & 4.254 & 4.778 & 5.259 & 5.740 & 6.263 \\
\hline
\end{tabular}

Table 3: Expectation of time $T$ until a proportion, $\rho$, of the population has not yet adopted the technology for different values $\rho$.

\section{Conclusions}

In this paper, we have provided a closed expression for the first probability density function (1-p.d.f.) of the solution stochastic process of the random SI-model (3), where uncertainty has been considered in the initial condition, $P_{0}$, and the nonlinear diffusion coefficient, $\beta$, regardless their probability distributions. As a consequence, we have shown that important probabilistic information can be computed from the 1-p.d.f., namely, the mean and variance functions, confidence intervals and the distribution of time until a given proportion of susceptibles remains in the population. The study has been based on the Random Variable Transformation method. The theoretical development has been applied to a pair of illustrative examples. In particular, the second example takes advantage of the obtained theoretical results to modelling the diffusion of a new technology based on real data. Finally, we want to point out that an important feature of our approach is that it can be extended to study other significant epidemiological models such 


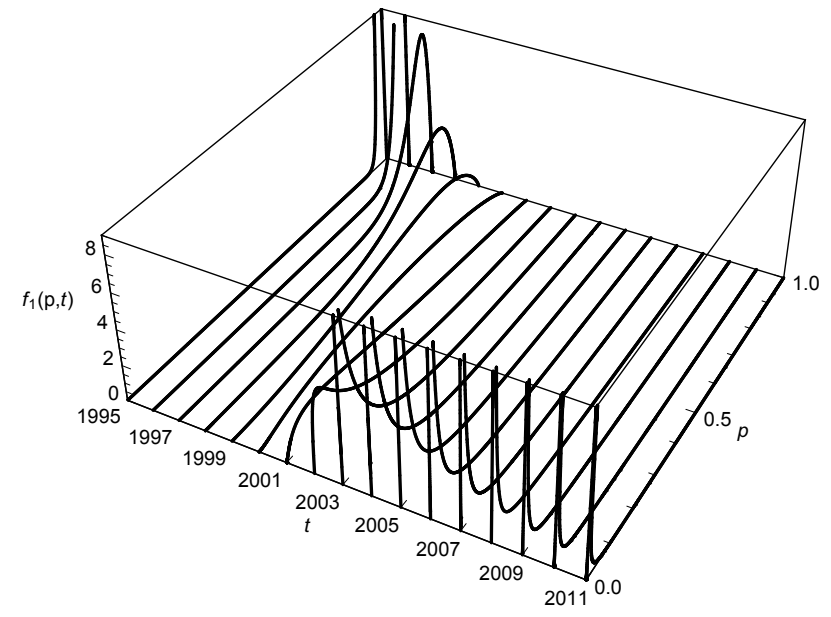

Figure 4: Plot of $f_{1}(p, t)$ in the Example 2 at the years 1995, 1996, , 2011 (corresponding to the solid lines).
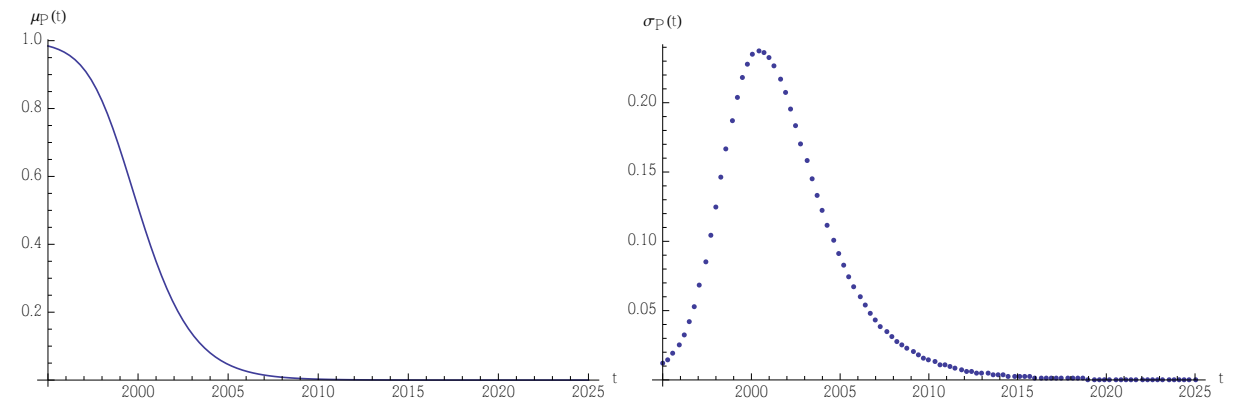

Figure 5: Plot of expectation function (left) and standard deviation function (right) in Example 2. 


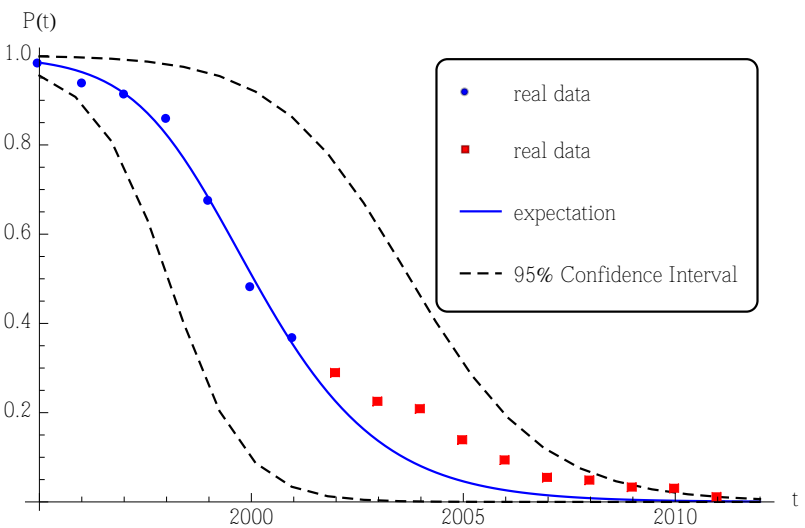

Figure 6: Expectation (solid line) and 95\% confidence intervals (dotted lines) in the Example 2. The real data used to fit the parameters $a, b, \lambda, \tau$ given by (47) are plotted in red color, whereas blue points correspond to the rest of the real data employed to validate the model.

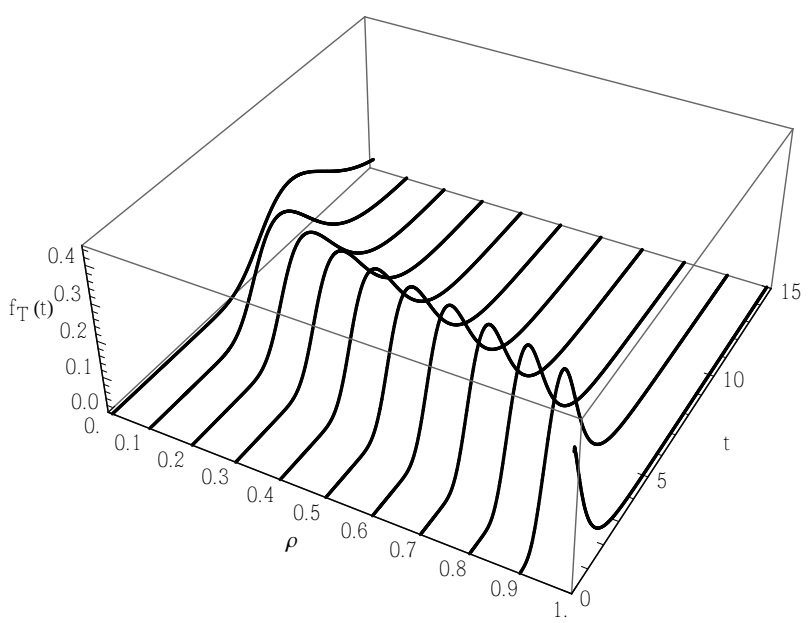

Figure 7: Plot of the p.d.f. of the time $T$ until a proportion $\rho$ of susceptibles remains in the population in Example 2 . 
as the SIS/SIR/SIRS-types models, etc., as well as other nonlinear models based on differential equations which often appear in other scientific areas.

\section{Acknowledgements}

This work has been partially supported by the Ministerio de Economía y Competitividad grants MTM2013-41765-P and TRA2012-36932.

\section{Conflict of Interest Statement}

The authors declare that there is no conflict of interests regarding the publication of this article.

[1] T. C. Gard, Introduction to Stochastic Differential Equations, Marcel Dekker, New York, 1988.

[2] P. E. Kloeden, E. Platen, Numerical Solution of Stochastic Differential Equations, 3rd Edition, Vol. 23 of Applications of Mathematics: Stochastic Modelling and Applied Probability, Springer, New York, 1999.

[3] T. T. Soong, Random Differential Equations in Science and Engineering, Academic Press, New York, 1973.

[4] R. G. Ghanem, P. D. Spanos, Stochastic Finite Elements: A Spectral Approach, Courier Dover Publ., New York, 2003.

[5] A. Papoulis, S. U. Pillai, Probability, Random Variables and Stochastic Processes, 4th Edition, McGraw-Hill, New York, 2002.

[6] M. A. El-Tawil, W. El-Tahan, A. Hussein, Using fem-rvt technique for solving a randomly excited ordinary differential equation with a random operator, Applied Mathematics and Computation 187 (2) (2007) 856-867. doi:10.16/j.amc.2006.08.164.

[7] M. C. Casabán, J. C. Cortés, J. V. Romero, M. D. Roselló, Determining the first probability density function of linear random initial value problems by the random variable transformation (r.v.t.) technique: A comprehensive study, Abstract and Applied Analysis 2014-ID248512 (2014) 1-25. doi:10.1155/2013/248512.

[8] A. Hussein, M. M. Selim, Solution of the stochastic transport equation of neutral particles with anisotropic scattering using RVT technique, Applied Mathematics and Computation 213 (1) (2009) $250-261$. doi:10.1016/j.amc.2009.03.016.

[9] A. Hussein, M. M. Selim, A developed solution of the stochastic Milne problem using probabilistic transformations, Applied Mathematics and Computation 216 (10) (2009) 2910-2919. doi:10.1016/j.amc.2010.04.003.

[10] A. Hussein, M. M. Selim, Solution of the stochastic radiative transfer equation with Rayleigh scattering using RVT technique, Applied Mathematics and Computation 218 (13) (2012) 7193-7203. doi:10.1016/j.amc.2011.12.088.

[11] L. T. Santos, F. A. Dorini, M. C. C. Cunha, The probability density function to the random linear transport equation, Applied Mathematics and Computation 216 (5) (2010) 1524-1530. doi:10.16/j.amc.2010.03.001.

[12] M. C. Casabán, J. C. Cortés, J. V. Romero, M. D. Roselló, Probabilistic solution of random homogeneous linear second-order difference equations, Applied Mathematics Letters 34 (2) (2014) $27-32$. doi:10.1016/j.aml.2014.03.0102.

[13] M. A. El-Tawil, The approximate solutions of some stochastic differential equations using transformations, Applied Mathematics and Computation 164 (1) (2005) 167-178. doi:10.1016/j.amc.2004.04.062.

[14] N. T. J. Bailey, The Mathematical Theory of Infectious Diseases, 2nd Edition, Hafner Press, New York, 1975.

[15] R. M. Anderson, Population Dynamics of Infectious Diseases, Chapman \& Hall, London, 1982.

[16] R. M. Anderson, R. M. May, Infectious Diseases of Humans, Oxford University, London, 1992.

[17] H. W. Hethcote, The mathematics of infectious diseases, SIAM Review 42 (4) (2000) 599-653 doi:http://www.siam.org/journals/sirev/42-4/37190.htnil.

[18] F. Brauer, C. Castillo-Chávez, Mathematical Models in Population Biology and Epidemiology, Springer, New York, 2001.

[19] B. Kegan, R. W. West, Modeling the simple epidemic with deterministic differential equations and random initial conditions, Mathematical Biosciences 195 (2) (2005) 179-193. doi:10.1016/j.mbs.2005.02.004.

[20] E. Rogers, Diffusion of Innovations, Free Press, New York, 2003.

[21] D. Zhang, A. Ntoko, Computational Methods in Decision-Making, Economics and Finance, Vol. 74 of Applied Optimization, Springer, New York, 2002, Ch. Mathematical Model of Technology Diffusion in Developing Countries, pp. 526-539.

[22] G. Casella, R. Berger, Statistical Inference, Brooks/Cole, New York, 2002. 
[23] CMT (Ed.), Anual Report of the Spanish National Committee of Telecommunications 2010-2012, CMT, Madrid, 2013.

[24] J. A. Nelder, R. Mead, A simplex method for function minimization, Computer Journal 7 (1) (1964) $308-313$. 